\title{
METODOLOGÍA DE SIMULACIÓN: SIMULADOR BABY REAL CARE II EN EL PROCESO DE ENSENAANZA APRENDIZAJE
} Simulation Methodology: Baby Real Care II Simulator in the Teaching Learning Process

\author{
Cleofé Genoveva Alvites-Huamaní ${ }^{1}$
}

Universidad Alas Peruanas, Perú

\section{RESUMEN}

La investigación fue de tipo experimental, con diseño cuasiexperimental, posprueba y grupo de control. Al grupo experimental se le aplicó la metodología de simulación a través del simulador Baby Real; al grupo de control se le mantuvo con el método tradicional utilizando un placebo (muñeco). La muestra estuvo conformada por 32 estudiantes de pregrado, entre 22 y 29 años de edad. Para contrastar la hipótesis se aplicó a ambos grupos el Cuestionario para el Usuario, conformado de 20 ítems. Los resultados se analizaron con el estadístico de Razón Critica de diferencia de porcentajes: en el área de semejanza con la realidad se obtuvo entre 75\% y $87,5 \%$, considerando al simulador como un nińo físicamente similar a un humano, válido para la enseñanza; en el área de motivación con el curso entre, $87,5 \%$ y $93,75 \%$; en el área herramienta de apoyo a la enseńanza, un $87,5 \%$; en el área de utilidad de la herramienta, un $100 \%$, siendo esta área la más alta significativamente, en la cual se consideró que los docentes deben utilizar herramientas de apoyo de simulación para sus clases teóricas. Asimismo, ambos grupos manifestaron que no habían utilizado en otro curso la metodología de simulación. En el área de sentimientos de aproximación a los contenidos de la herramienta, a un 50\% les gustó la simulación. Se concluyó que de acuerdo al nivel de intervalos existe una mayor significancia $(0,75 \%)$ en la aplicación de la metodología de simulación considerada "eficiente" como método de enseñanza; por lo que se aceptó la hipótesis planteada.

Palabras Clave: Metodología de simulación, simuladores, tecnologías, educación

\section{Abstract}

The research was experimental, quasi-experimental design with, post-test and control group. The simulation methodology by means of the Baby Real simulator was applied to the experimental group; the control group was kept to the traditional method using a placebo (dummy). The sample consisted of 32 undergraduate students, between 22 and 29 years old. To test the hypothesis both groups took the Questionnaire for User, consisting of 20 items. The results were analyzed with statistical Critique of Reason percentage difference: in the area of resemblance to reality we obtained between $75 \%$ and $87,5 \%$, considering the simulator as a child physically similar to a human, valid for teaching; in the area of motivation to the course we had between $87,5 \%$ and $93,75 \%$; in the area of support to education, $87,5 \%$; and in the area of use of the tool, we got $100 \%$, being this area the highest one, in which it was considered that teachers should use simulation tools to support their lectures. Also, both groups reported they had no used simulation methodology in other course.

\footnotetext{
1Directora, asesora y revisora de Tesis de la Unidad de Investigación de Post Grado de diversas universidades, Coordinadora de la División de Investigación y Extensión Científica Tecnológica de la Dirección Universitaria de Educación a Distancia de la Universidad Alas Peruanas, adscrita al Directorio Nacional de Investigadores e Innovadores.cleoalvitesh@gmail.com, c_alvites@uap.edu.pe.
} 
Feelings in the approach area to the contents of the tool, $50 \%$ liked the simulation. It was concluded that according to the level of intervals there is a greater significance $(0.75 \%)$ in the application of simulation methodology considered "efficient" as a teaching method; so the hypothesis was accepted.

Keywords: Simulation methodology, simulator, technologies, education.

\section{INTRODUCCIÓN}

En la enseñanza desde la educación básica, hasta la superior, se busca métodos, estrategias y metodologías adecuadas que permitan desarrollar competencias, habilidades, destrezas, así como la gestión de conocimiento utilizando diversos medios, materiales y herramientas tecnológicas. Desde esta perspectiva el presente estudio tiene como objetivo general analizar las diferencias significativas como resultado de la metodología de simulación a través del Simulador Baby Real Care II, aplicada en el curso de estimulación temprana y cuidados y protección del infante, con relación a la metodología tradicional utilizando un muñeco (placebo) en estudiantes de pregrado.

Figura 1. Simulador "Baby Real Care II". Realityworks. Live it. Learn it

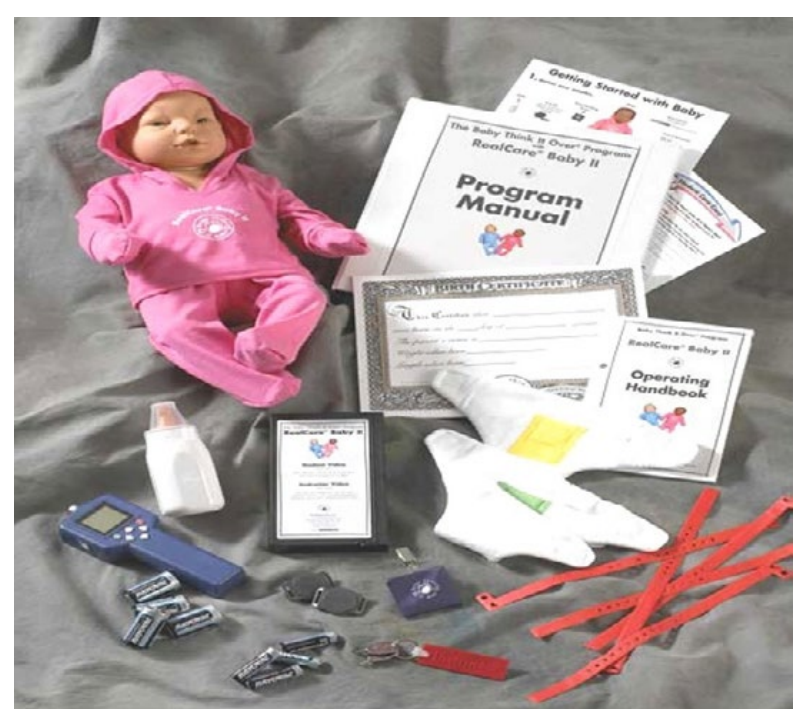

Como objetivos específicos se consideraron: (1) describir la semejanza con la realidad; (2) verificar la motivación hacia los cursos de Estimulación temprana y Cuidados y protección del infante, (3) evaluar como herramienta de apoyo el simulador, (4) analizar la utilidad de la herramienta, (5) identificar los sentimientos de aproximación a los contenidos de la herramienta; y (6) mencionar las sugerencias para mejoras del simulador. Y como hipótesis general: Difiere significativamente la metodología de simulación a través del simulador Baby Real Care II, aplicada en el curso de estimulación temprana y Cuidados y protección del infante, respecto de la metodología tradicional utilizando un muńeco (placebo) en estudiantes de pregrado de educación.

\section{Antecedentes}

Cano, Tamayo, Pardo y Martínez (2010) refieren en su investigación realizada para evaluar la simulación clínica como herramienta de aprendizaje y adquisición de competencias en el manejo de situaciones críticas a 20 estudiantes residentes por un lapso de dos ańos. Recolectaron los datos a través de un cuestionario que evalúa las competencias relacionadas a las habilidades técnicas y de conocimientos que iban desarrollando como parte de su formación. Obtuvieron como resultado que el $85 \%$ de los residentes habían mejorado sus conocimientos; el 100\% afirmaron estar más seguros para la toma de decisiones y mejoraron sus habilidades de comunicación y la capacidad de trabajar en equipo; el $85 \%$ obtuvieron una mejoría en su capacidad para gestionar el estrés y organizar el trabajo y el $80 \%$ mejoraron su capacidad de liderazgo. Cano et al concluyeron que la simulación clínica es una herramienta útil y permite la adquisición de habilidades técnicas, de conocimientos y para el desarrollo de diversas competencias.

Marqués (2011), en su investigación sobre el uso de las Tecnologías de la Información y Comunicación (Tics) en la práctica docente, refiere que al utilizar los recursos tecnológicos en el proceso de aprendizaje-enseñanza hay un aumento de la 
atención y motivación del 100\%; estas facilitan la comprensión en un 98\%; aumentan el acceso a recursos para comentar y compartir entre el alumnado y docentes en un $92 \%$. Permite el logro de los objetivos en un $90 \%$. Facilitan la contextualización de las actividades en un $82 \%$. El $91 \%$ de los docentes aseveran que los estudiantes mejoran su aprendizaje con las actividades realizadas con Tics y están motivados a ejecutarlas con mayor ímpetu.

Ros y Conesa (2013), en su estudio sobre adquisición de competencias a través de la simulación, comprobaron que el $90 \%$ de los estudiantes consideran que la técnica de aprendizaje con la simulación les ha permitido adquirir las competencias específicas asignadas. Además enfatiza que los profesores y tutores necesitan también un entrenamiento específico en el manejo, diseño e implementación de este tipo de acciones formativas y el esfuerzo que se requiere para formarlos en esta nueva metodología.

Por su parte, Garcia-Carbonell, Watts y Andreu (2012), en su estudio sobre la simulación como experiencia en el aprendizaje de lengua inglesa, comprobaron que los dos grupos experimentales postratamiento del uso de la simulación habían mejorado significativamente sus destrezas en comprensión lectora y oral, a diferencia de los tres grupos control. Los resultados totales obtenidos pre y postratamiento evidenciaron mejoras en ambos grupos; pero el grupo experimental presentó mayor significancia $\mathrm{p}=0,000$ en comprensión oral, expresión escrita, comprensión lectora y gramática, al utilizar la simulación. Concluyeron que la simulación es una herramienta con potencial extraordinario para la enseñanza-aprendizaje.

Toledo y Camero (2010), en su estudio sobre la simulación-juego en un curso de biología celular, evaluaron los resultados de la aplicación de la estrategia utilizando una prueba de conocimientos y las reflexiones realizadas por los estudiantes. Se comprobó que la simulación tuvo una aceptación de $86,78 \%$. En cuanto al rendimiento académico hubo un incremento del $56 \%$ de los grupos que experimentaron con la simulación, a diferencia de las calificaciones de los grupos en los que no se aplicó la simulación (48\%). Con relación a las reflexiones de la metodología de simulación, en este mismo estudio el autor indica que los participantes han considerado significantes a ciertas cualidades de la simulación: original, eficaz, amena, atractiva y excelente. En cuanto a los atributos pedagógicos, consideraron contenidos bien manejados; como recurso instruccional: creativo, apropiado, didáctico y comprensible, aumenta el interés por el tema.

\section{METODOLOGÍA DE SIMULACIÓN}

Aguirre (2012) señala que la simulación como técnica a través del uso de simuladores es referirse a las tecnologías que permiten recrear un ambiente $\mathrm{u}$ objeto en el que puede repetirse y aprender un procedimiento cada vez que lo requiera uno de los participantes. Este procedimiento permite reproducir objetos y entornos reales, debido a que por inconvenientes de recurso o por seguridad, el estudiante no pueda realizar dicha actividad en su ambiente natural. Para Corvetto et al (2013), la simulación crea un ambiente ideal para la educación, ya que las actividades pueden diseñarse con la finalidad de que el participante pueda predecir y reproducir situaciones a las que se enfrentará en su campo profesional, al mismo tiempo que proporciona el entrenamiento sistemático y repetido de habilidades prácticas y competencias, permitiéndose el estudiante se equivoque y aprenda del error. Es decir, el proceso de aprendizaje se basa en la práctica y la reflexión, brinda una mayor transferencia de conocimientos al integrar teoría y práctica. Al mismo tiempo sirve como herramienta de evaluación.

Andreu, García y Mollar (2005) resaltan que con la simulación los estudiantes experimentan y se recrean con la realidad; participan activamente en tareas ya programadas con un objetivo específico, que deben concretizar. Esta metodología brinda la oportunidad de situar al estudiante en un contexto que imite algún aspecto de la realidad, generándose en ese ambiente situaciones problemáticas o reproductivas similares a las que deberá enfrentar profesionalmente. El propósito es lograr en los futuros estudiantes el desarrollo de un conjunto de competencias, habilidades y 
destrezas que les permitan alcanzar el perfil profesional deseado (Alvites-Huamaní, 2010).

Aguirre (2012) menciona que la simulación como herramienta para la enseñanza permite controlar sistemáticamente el horario de la práctica, la presentación de la información, la introducción o supresión de las distracciones del entorno, brindando al estudiante un ambiente seguro y controlado. Además refiere que la simulación como estrategia didáctica permite hacer más eficiente los procesos de aprendizaje; contribuye a mejorar la calidad de la educación, por lo que representa una revolución en la educación. Pales y Gomar (2010) indican que la simulación es cualquier actividad docente que utilice simuladores con la finalidad de estimular y favorecer el aprendizaje. (p. 149).

Para Prensky (2010), citado por Gisbert (2013), el valor formativo de las simulaciones se observa en la evidencia de que los procesos de aprendizaje realmente efectivos permiten probar, construir, experimentar, tomar decisiones, resolver problemas, es decir, requieren una posición activa del estudiante. Son estas situaciones de aprendizaje con las que se adquiere competencias y se genera conocimiento con más facilidad.

Ruiz (2012), citado por Alvites-Huamaní (2014), señala que la simulación es una herramienta de aprendizaje cuyo objetivo principal es la adquisición de competencias a través del entrenamiento en un ambiente u objeto lo más parecido posible al real. El estudiante puede realizar repeticiones todas las veces que desee para lograr el dominio del procedimiento o técnica. Esto permite que el margen de error humano sea reducido. Desde esta perspectiva, los avances tecnológicos en la educación superior constituyen un elemento de incorporación estratégica para mejorar los procesos de enseńanza aprendizaje.

\section{MATERIALES Y MÉTODO}

\section{Participantes}

Se trabajó con una muestra no probabilística de grupos intactos, constituida por 32 estudiantes de pregrado de una universidad privada de Perú, todas inscritas en el curso de Estimulación Temprana. Todos los participantes son de sexo femenino, agrupadas en dos secciones de 16 estudiantes cada una, con edad entre los 19 y 28 años y de un nivel socioeconómico medio a bajo.

\begin{tabular}{llc}
\hline Sección & \multicolumn{1}{c}{ Turno } & Cantidad \\
\hline 1 & Mañana & 16 \\
2 & Noche & 16 \\
\hline
\end{tabular}

Tabla 1. Descripción de la muestra

\section{Instrumento}

Se elaboró el cuestionario para el usuario del Baby Real Care II, el cual constaba de 20 ítems.

\begin{tabular}{|c|c|c|c|}
\hline Dimensión & Indicador & Índice & Instrumento \\
\hline $\begin{array}{l}\text { Semejanza } \\
\text { con la realidad }\end{array}$ & $\begin{array}{l}\text { Apariencia } \\
\text { Horarios } \\
\text { Cuidados } \\
\text { Sonidos }\end{array}$ & 1 & \\
\hline $\begin{array}{l}\text { Motivación con } \\
\text { los cursos }\end{array}$ & $\begin{array}{l}\text { Motivación } \\
\text { Práctica } \\
\text { Seguridad } \\
\text { Ensayo }\end{array}$ & $\begin{array}{l}2 \\
3 \\
4 \\
5\end{array}$ & \\
\hline $\begin{array}{l}\text { Herramienta } \\
\text { de apoyo }\end{array}$ & $\begin{array}{l}\text { Herramienta } \\
\text { Software de } \\
\text { Registro } \\
\text { Evaluación } \\
\text { Manejar } \\
\text { Identificación }\end{array}$ & $\begin{array}{l}1 \\
2\end{array}$ & $\begin{array}{l}\text { Cuestiona- } \\
\text { rio para el } \\
\text { usuario del } \\
\text { Baby Real } \\
\text { Care II }\end{array}$ \\
\hline $\begin{array}{l}\text { Utilidad de la } \\
\text { herramienta }\end{array}$ & $\begin{array}{l}\text { Simulación } \\
\text { Herramienta } \\
\text { apoyo }\end{array}$ & & \\
\hline $\begin{array}{l}\text { Sentimientos } \\
\text { de aproxi- } \\
\text { mación a los } \\
\text { contenidos de } \\
\text { la herramienta }\end{array}$ & $\begin{array}{l}\text { Gusto } \\
\text { No gusto }\end{array}$ & & \\
\hline $\begin{array}{l}\text { Sugerencias } \\
\text { para mejoras }\end{array}$ & $\begin{array}{l}\text { Recomenda- } \\
\text { ciones }\end{array}$ & & \\
\hline
\end{tabular}

Tabla 2. Alumnos: composición estratificada de la muestra

Se evaluaron las áreas: semejanza con la realidad (4 ítems), motivación con el curso (5 ítems), he- 
rramienta de apoyo (6 ítems), utilidad de la herramienta ( 2 ítems), sentimientos de aproximación a los contenidos de la herramienta ( 2 ítems) y sugerencias para mejoras (1 ítem). La validez del cuestionario se verificó mediante el método de jueces o expertos, con un valor de Ji Cuadrado del 0,05. La confiabilidad se determinó mediante el método de test-retest, para lo cual se aplicó el cuestionario dos veces, con un intervalo de 3 semanas entre ambas aplicaciones; con ambos resultados se calculó el coeficiente de correlación de Spearman, siendo un 0,82 de confiabilidad del instrumento.

\section{Diseño}

El estudio es de tipo experimental con diseño cuasi experimental, posprueba y grupo control. $\mathrm{Al}$ grupo experimental se aplicó la metodología de simulación usando el simulador Baby Real II como herramienta de apoyo al proceso de enseñanza; al de control, la metodología tradicional utilizando un placebo (muñeco). A los dos grupos se les brindó simultáneamente los mismos contenidos.

\begin{tabular}{llc}
\hline Grupo & Experimento & Posprueba \\
\hline$G_{1}$ & $\mathrm{X}$ & $\mathrm{O}_{1}$ \\
$\mathrm{G}_{2}$ & - & $\mathrm{O}_{2}$ \\
\hline
\end{tabular}

Donde: $\mathrm{G}_{1}=$ grupo experimental; $\mathrm{G}_{2}=$ Grupo de control; $\mathrm{X}=$ estímulo; - = ausencia de estímulo

Tabla 3. Diagrama del diseño.

\section{Procedimiento}

El curso de estimulación temprana se realizó en un semestre académico de 5 meses y medio. Las sesiones se desarrollaron semanalmente, a razón de 4 horas. Se trabajó con dos grupos designados por dirección académica en dos horarios distintos, un grupo en la mañana y otro en la noche. Durante el semestre se impartió en ambos grupos (control y experimental) los mismos contenidos en clase. A partir del segundo mes se trabajó con el simulador Baby Real Care II con el grupo experimental y con el grupo control un muñeco (placebo); simultáneamente se realizaba la teoría y la práctica con el simulador y el placebo. Al finalizar el curso se aplicó a ambos grupos el cuestionario de usuario del Baby Real Care II, para contrastar las hipótesis. Los datos recolectados se procesaron con el programa estadístico SPSS v.17. Los resultados se presentan en tablas y gráficos estadísticos. En el análisis se utilizó la razón crítica de diferencias de porcentaje, con la finalidad de evaluar la aceptación o rechazo de la hipótesis.

\section{RESULTADOS}

\section{Área semejanza con la realidad}

La tabla 4 muestra los resultados con el análisis de la razón crítica de diferencias de porcentajes de respuesta de acuerdo a cada pregunta para el área de semejanza con la realidad.

\begin{tabular}{|c|c|c|c|c|c|c|c|c|}
\hline \multirow{2}{*}{ Ítem } & \multicolumn{2}{|c|}{ De acuerdo } & \multicolumn{2}{|c|}{ De acuerdo } & \multirow[b]{2}{*}{ Dif. } & \multirow[b]{2}{*}{ EE } & \multirow[b]{2}{*}{$\mathrm{RC}$} & \\
\hline & $\begin{array}{l}\text { G. } \\
\text { Ex. }\end{array}$ & $\%$ & $\begin{array}{c}\text { G. } \\
\text { Con. }\end{array}$ & $\%$ & & & & \\
\hline 1 & 12 & 75.00 & 3 & 19.0 & 56 & 15 & 4 & * \\
\hline 2 & 14 & 87.50 & 0 & 0 & 88 & 8 & 11 & * \\
\hline $3 a$ & 13 & 81.25 & 0 & 0 & 81 & 10 & 8 & * \\
\hline $3 b$ & 12 & 75.00 & 0 & 0 & 75 & 11 & 7 & * \\
\hline $3 c$ & 11 & 68.75 & 4 & 25.0 & 44 & 16 & 3 & * \\
\hline $3 d$ & 12 & 75.00 & 0 & 0 & 75 & 11 & 7 & * \\
\hline $3 e$ & 12 & 75.00 & 0 & 0 & 75 & 11 & 7 & * \\
\hline $4 a$ & 14 & 87.50 & 0 & 0 & 88 & 8 & 11 & * \\
\hline $4 b$ & 13 & 81.25 & 0 & 0 & 81 & 10 & 8 & * \\
\hline $4 c$ & 11 & 68.75 & 0 & 0 & 69 & 12 & 6 & * \\
\hline $4 d$ & 14 & 87.50 & 0 & 0 & 88 & 8 & 11 & * \\
\hline $4 e$ & 14 & 87.50 & 6 & 38.0 & 50 & 15 & 3 & * \\
\hline $4 f$ & 8 & 50.00 & 0 & 0 & 50 & 13 & 4 & * \\
\hline Total & 160 & 76.92 & 13 & 6.0 & 71 & 12 & 6 & \\
\hline
\end{tabular}

$\geq$ Nivel de .01 de significancia

Tabla 4. Semejanza con la Realidad

Se puede observar que, con relación al área de semejanza con la realidad, el $75 \%$ del grupo experimental manifestó estar de acuerdo en que la apariencia física del simulador Baby Real es similar a un bebe real (ítem1); en el grupo control apenas el 19\%. El 87,5\% del grupo experimental manifestó estar de acuerdo en que los horarios establecidos del simulador son similares a un bebé real (ítem 2); en contraste con el grupo de control, que no estuvo de acuerdo (0\%). El 81,25\% de participantes del grupo experimental estuvieron de acuerdo en que la forma de cuidado del simulador se asemejó a un bebé real, en calmar su llanto (ítem 3a). Un 75\% manifestó que en 
alimentarlo (ítem 3b); el 68,75\%, en cambiarle el pañal (ítem 3c); el 75\%, en hacerlo eructar (ítem 3d); el 75\%, en arrullarlo (ítem 3e). El 75\% del grupo experimental manifestó estar de acuerdo con la semejanza del cuidado de un bebé real al cuidar al simulador; a diferencia del grupo control, en el que solo el $25 \%$ estuvo de acuerdo en que el cuidado del muñeco (placebo) se asemejó a un bebe real al cambiar el pañal. El $87,5 \%$ estuvo de acuerdo en que los sonidos emitidos por el simulador se asemejan a un bebe real, respirar mientras duerme (ítem4a). El 81,25\%, en comer (ítem 4b); el $68.75 \%$ en reír al arrullarlo (ítem 4c); el 87.5\%, en eructar (ítem 4d); el 87.5\%, en llorar (ítem 4e); El 50\% del grupo experimental estuvo de acuerdo en la semejanza de los sonidos emitidos por el simulador con un bebé real al toser (ítem 4f). En cambio, el 38\% del grupo de control manifestó estar de acuerdo en que los sonidos emitidos por el muńeco (placebo) en el llanto se asemejaron a un bebe real.

\section{Área motivación con el curso}

La tabla 5 muestra los resultados del análisis de la razón crítica de diferencias de porcentajes respecto de las preguntas para el área de Motivación con el curso.

\begin{tabular}{|c|c|c|c|c|c|c|c|c|}
\hline \multirow{2}{*}{ Ítem } & \multicolumn{2}{|c|}{ De acuerdo } & \multicolumn{2}{|c|}{ De acuerdo } & \multirow[b]{2}{*}{ Dif. } & \multirow[b]{2}{*}{ EE } & \multirow[b]{2}{*}{$\mathrm{RC}$} & \\
\hline & $\begin{array}{l}\text { G. } \\
\text { Ex. }\end{array}$ & $\%$ & $\begin{array}{c}\text { G. } \\
\text { Con. }\end{array}$ & $\%$ & & & & \\
\hline 5 & 13 & 81.25 & 2 & 13.0 & 69 & 13 & 5 & * \\
\hline 6 & 15 & 93.75 & 2 & 13.0 & 81 & 10 & 8 & * \\
\hline $7 a$ & 11 & 68.75 & 2 & 13.0 & 56 & 14 & 4 & . \\
\hline $7 b$ & 15 & 93.75 & 2 & 13.0 & 81 & 10 & 8 & $\wedge$ \\
\hline $7 c$ & 14 & 87.5 & 2 & 13.0 & 75 & 12 & 6 & . \\
\hline $7 d$ & 11 & 68.75 & 2 & 13.0 & 56 & 14 & 4 & 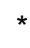 \\
\hline $7 e$ & 14 & 87.5 & 2 & 13.0 & 75 & 12 & 6 & ^ \\
\hline 8 & 15 & 93.75 & 0 & 0.0 & 94 & 6 & 15 & * \\
\hline 9 & 14 & 87.5 & 0 & 0.0 & 88 & 8 & 11 & * \\
\hline Total & 122 & 84.72 & 14 & 9.72 & 75 & 12 & 6 & * \\
\hline
\end{tabular}

$\geq$ Nivel de .01 de significancia

Tabla 5. Motivación con el curso

Se observa que el $81,25 \%$ del grupo experimental estuvo de acuerdo en que el uso del simulador Baby Real les hizo sentir más motivadas a aprender la teoría del curso de estimulación temprana (ítem 5), a diferencia del grupo de control, en el que solo el 13\% estuvo de acuerdo. El 93,75\% de las alumnas del grupo experimental manifestaron que se sintieron más motivas a aprender la teoría del curso (ítem 6), a diferencia del grupo de control en el que solo un $13 \%$ se sintieron motivadas por el muñeco (placebo). El 68,75\% del grupo experimental manifestó estar de acuerdo con que el tener al simulador a su cuidado les sirvió como herramienta de apoyo para poner en práctica sus conocimientos sobre las técnicas de masajes de estimulación temprana (ítem 7), a diferencia del grupo de control que indicaron estar de acuerdo que un $13 \%$ con que el placebo les sirvió como herramienta de apoyo. El 97,75\% del grupo experimental mencionó estar de acuerdo en que tener a su cuidado al simulador les sirvió como herramienta de apoyo para poner en práctica sus conocimientos teóricos sobre cómo cuidar y proteger a un infante (ítem 7b); esta misma impresión la tuvieron un $13 \%$ del grupo de control. El $87,5 \%$ del grupo experimental manifestó estar de acuerdo con que tener al simulador Baby Real a su cuidado les sirvió como herramienta de apoyo para vencer temores de cuidar y estimular a bebes reales (ítem 7c); en el grupo control solo el 13\% estuvo de acuerdo con esto. El 68,75\% del grupo experimental estuvo de acuerdo en que tener al simulador a su cuidado les sirvió para tener mayor seguridad para interactuar con bebes reales (ítem 7d); en cambio solo el 13\% del grupo de control manifestó lo mismo. El 87,5\% del grupo de control estuvo de acuerdo en que cuidar al simulador les sirvió de apoyo para vivenciar una simulación con un bebe virtual (ítem 7e); en el grupo control solo el 13\% manifestaron lo mismo.

El 93,75\% de las estudiantes del grupo experimental manifestaron estar de acuerdo en que el ensayo-práctica simulada de las técnicas y masajes de estimulación con el simulador les sirvió para hacerlo mejor en el taller de estimulación temprana con niños reales de 0-2 (ítem 8); por el contrario, el grupo control indicó que el muñeco (placebo) no les sirvió para el taller mencionado. El 87,5\% de las alumnas del grupo experimental manifestaron que el ensayo - práctica simulada del cuidado y protección de un bebé con el Baby Real les sirvió para hacerlo mejor en las aulas de cuna con niños reales de 1 a 6 meses (ítem 9); a 
diferencia del grupo control, que indicó que el muñeco (placebo) no le sirvió para desempeñarse mejor en aula con nińos reales.

\section{Área herramienta de apoyo}

En la tabla 6 se muestran los resultados con el análisis de la razón crítica de diferencias de porcentajes de respuesta a cada pregunta para el área de herramienta de apoyo.

\begin{tabular}{|c|c|c|c|c|c|c|c|c|}
\hline \multirow[b]{2}{*}{ Ítem } & \multicolumn{2}{|c|}{ De acuerdo } & \multicolumn{2}{|c|}{ De acuerdo } & \multirow[b]{2}{*}{ Dif. } & \multirow[b]{2}{*}{$\mathrm{EE}$} & \multirow[b]{2}{*}{$\mathrm{RC}$} & \\
\hline & $\begin{array}{l}\text { G. } \\
\text { Ex. }\end{array}$ & $\%$ & $\begin{array}{c}\text { G. } \\
\text { Con. }\end{array}$ & $\%$ & & & & \\
\hline 10 & 14 & 87.5 & 0 & 0 & 88 & 8 & 11 & * \\
\hline 11 & 8 & 50.0 & 0 & 0 & 50 & 13 & 4 & * \\
\hline 12 & 14 & 87.5 & 0 & 0 & 88 & 8 & 11 & * \\
\hline 13 & 14 & 87.5 & 0 & 0 & 88 & 8 & 11 & * \\
\hline 14 & 14 & 87.5 & 0 & 0 & 88 & 8 & 11 & * \\
\hline 15 & 14 & 87.5 & 0 & 0 & 88 & 8 & 11 & * \\
\hline Total & 78 & 81.25 & 0 & 0 & 81 & 10 & 8 & * \\
\hline
\end{tabular}

$\geq$ Nivel de .01 de significancia

Tabla 6. Herramienta de apoyo

El 87.5\% del grupo experimental manifestó estar de acuerdo en que el simulador Baby Real es válido como herramienta de apoyo al aprendizaje para el curso de cuidados y protección del infante" (ítem 10); a diferencia del grupo de control, en el que un $0 \%$ mencionó estar de acuerdo en que el muñeco (placebo) es válido como herramienta de apoyo. El 50\% de las estudiantes del grupo experimental manifestaron que el simulador es válido como herramienta de apoyo al aprendizaje para el curso de estimulación temprana (ítem 11); a diferencia del grupo control que indicó que el muñeco (placebo) no es válido como herramienta de apoyo para el curso mencionado. El 87,5\% de las estudiantes del grupo experimental manifestaron que es correcto que el simulador Baby Real cuente con un software de registro del cuidado que le dio la persona que lo tuvo a su cargo (ítem 12); a diferencia del grupo de control que no indicó nada, ya que el muñeco (placebo) no cuenta con este software. El 87,5\% del grupo experimental manifestaron estar de acuerdo que la evaluación que emite el software de la persona que tuvo a cargo al simulador es válida (ítem 13), a diferencia del grupo control que no indicó nada, ya que el muñeco (placebo) no cuenta con esta evaluación. Al 87,5\% del grupo experimental les pareció fácil manejar, utilizar y cuidar al simulador (ítem 14), a diferencia del grupo de control que no indicó nada. Al 87,5\% del grupo experimental les pareció fácil usar el brazalete de identificación para atender al simulador Baby Real (ítem 15), a diferencia del grupo de control que no indicó nada porque el muñeco (placebo) no contaba con este dispositivo.

\section{Utilidad de la herramienta}

En la tabla 7 se muestran los resultados con el análisis de la razón crítica de diferencias de porcentajes de respuesta a cada pregunta para el área utilidad de la herramienta.

\begin{tabular}{|c|c|c|c|c|c|c|c|c|}
\hline \multirow[b]{2}{*}{ Ítem } & \multicolumn{2}{|c|}{ De acuerdo } & \multicolumn{2}{|c|}{ De acuerdo } & \multirow[b]{2}{*}{ Dif. } & \multirow[b]{2}{*}{ EE } & \multirow[b]{2}{*}{$\mathrm{RC}$} & \\
\hline & $\begin{array}{l}\text { G. } \\
\text { Ex. }\end{array}$ & $\%$ & $\begin{array}{c}\text { G. } \\
\text { Con. }\end{array}$ & $\%$ & & & & \\
\hline 16 & 0 & 0 & 0 & 0 & 0 & 0 & 0 & \\
\hline 17 & 16 & 100 & 0 & 0 & 100 & 0 & 100 & * \\
\hline Total & 16 & 50 & 0 & 0 & 50 & 13 & 101 & \\
\hline
\end{tabular}

$\geq$ Nivel de .01 de significancia

Tabla 7. Utilidad de la herramienta de apoyo

Ambos grupos (experimental y control) estuvieron de acuerdo en que en ningún otro curso de educación superior han utilizado la Metodología de simulación como apoyo a su aprendizaje antes de hacerlo en la vida real (ítem 16). El 100\% de las estudiantes del grupo experimental manifestó que después de su experiencia con Baby Real los educadores deben utilizar herramientas de apoyo de simulación para sus clases teóricas (ítem 17), a diferencia del grupo control que no indicó nada, ya que utilizaron un muñeco (placebo).

\section{Área sentimientos de aproximación a los conte- nidos de la herramienta}

La tabla 8 muestra los resultados con el análisis de la razón crítica de diferencias de porcentajes de respuesta de acuerdo a cada pregunta para el área de sentimientos de aproximación a los contenidos de la herramienta.

Respecto a la pregunta qué le gustó de la simulación con el Baby Real (ítem 18a), el 50\% del 
grupo experimental mencionó el calmar el llanto; un $25 \%$, darle las atenciones básicas y poder cuidarlo y protegerlo; a diferencia del grupo control que no indicó nada, ya que utilizaron un muñeco (placebo) que no tenía estas características. En el ítem 19a referido a qué no le gustó de la simulación con el Baby Real, el 43,75\% del grupo experimental manifestó que no es fácil hacerle masajes; el $75 \%$, no poder cambiarle la ropa fácilmente (ítem 19b); un 31,25\%, no poder interactuar emocionalmente con el simulador (ítem 19c), a diferencia del grupo de control que no indicó nada, ya que utilizaron un muńeco (placebo) que no tenía estas características.

\begin{tabular}{|c|c|c|c|c|c|c|c|}
\hline \multirow[b]{2}{*}{ Ítem } & \multicolumn{2}{|c|}{ De acuerdo } & \multicolumn{2}{|c|}{ De acuerdo } & \multirow[b]{2}{*}{ Dif. } & \multirow[b]{2}{*}{ EE } & \multirow[b]{2}{*}{$\mathrm{RC}$} \\
\hline & $\begin{array}{l}\text { G. } \\
\text { Ex. }\end{array}$ & $\%$ & $\begin{array}{l}\text { G. } \\
\text { Con. }\end{array}$ & $\%$ & & & \\
\hline $18 a$ & 4 & 25.00 & 0 & 0 & 25 & 11 & 2 \\
\hline $18 b$ & 8 & 50.00 & 0 & 0 & 50 & 13 & 4 \\
\hline $18 \mathrm{c}$ & 4 & 25.00 & 0 & 0 & 25 & 11 & 2 \\
\hline $19 a$ & 7 & 43.75 & 0 & 0 & 44 & 12 & 4 \\
\hline $19 b$ & 5 & 31.25 & 0 & 0 & 31 & 12 & 3 \\
\hline $19 c$ & 12 & 75.00 & 0 & 0 & 75 & 11 & 7 \\
\hline Total & 72 & 56.25 & 0 & 0 & 56 & 12 & 4 \\
\hline
\end{tabular}

$\geq$ Nivel de .01 de significancia

Tabla 8. Sentimientos de aproximación a los contenidos de la herramienta

\section{Área sugerencias para mejoras}

En la tabla 9 se muestran los resultados con el análisis de la razón crítica de diferencias de porcentajes de respuesta de acuerdo a cada pregunta para el área de sugerencias para mejoras.

\begin{tabular}{|c|c|c|c|c|c|c|c|}
\hline \multirow[b]{2}{*}{ Ítem } & \multicolumn{2}{|c|}{ De acuerdo } & \multicolumn{2}{|c|}{ De acuerdo } & \multirow[b]{2}{*}{ Dif. } & \multirow[b]{2}{*}{ EE } & \multirow[b]{2}{*}{$\mathrm{RC}$} \\
\hline & $\begin{array}{l}\text { G. } \\
\text { Ex. }\end{array}$ & $\%$ & $\begin{array}{c}\text { G. } \\
\text { Con. }\end{array}$ & $\%$ & & & \\
\hline $20 a$ & 9 & 56.25 & 0 & 0 & 56 & 12 & 5 \\
\hline $20 b$ & 9 & 56.25 & 0 & 0 & 56 & 12 & 5 \\
\hline $20 c$ & 12 & 75.00 & 0 & 0 & 75 & 11 & 7 \\
\hline Total & 30 & 62.50 & 0 & 0 & 63 & 12 & 5 \\
\hline
\end{tabular}

$\geq$ Nivel de .01 de significancia

Tabla 9. Sugerencias para mejoras

Se aprecia que no existen diferencias de porcentajes entre el grupo experimental y grupo control; solo el grupo experimental manifestó sugerencias para mejoras del Baby Real. Un 56,25\% sugirió que la piel del simulador sea suave (ítem 20a). El 56,25\% recomendó que los ojos se abran y cierren (ítem 20b). El $75 \%$ recomienda que se moje el pañal (ítem 20c), a diferencia del grupo de control que no manifestó sugerencias para el muñeco (placebo).

\section{DISCUSIÓN Y CONCLUSIONES}

Los resultados obtenidos con relación a la metodología de simulación a través del simulador Baby Real Care II han sido bastante significativos. Implicaron la aceptación de las hipótesis propuestas. En el área de semejanza con la realidad se obtuvo porcentajes entre $75 \%$ y $87,5 \%$ de aceptación respecto a que el simulador Baby Real es físicamente similar a un bebé y válido para el proceso de enseñanza y aprendizaje. Este resultado coincide con los hallazgos de Aguirre (2012), quien refiere que la simulación permite recrear objetos o contextos similares a los reales. Con relación al área de motivación con los cursos los porcentajes fueron entre $87,5 \%$ y $93,75 \%$; las estudiantes consideraron que el simulador es significativamente motivador para el aprendizaje de los cursos de Estimulación temprana y Cuidados y protección del infante. Esto corrobora lo descrito por Marqués (2011), para quien hay un aumento de la atención y motivación del $100 \%$, que implica la mejora del aprendizaje en los estudiantes.

En el área de herramienta de apoyo, los porcentajes obtenidos fueron de $87,5 \%$, aceptándose al simulador como una herramienta de apoyo en el proceso de aprendizaje-enseñanza. Dicho resultado se relaciona con los hallazgos de Cano et al (2010), quien al evaluar la simulación clínica como herramienta de aprendizaje y adquisición de competencias en el manejo de situaciones crítica concluyó que esta es una herramienta útil y permite la adquisición de habilidades técnicas, de conocimientos y el desarrollo de diversas competencias.

La evaluación al área de utilidad de la herramienta obtuvo el $100 \%$, siendo el área significativamente más alta. Las estudiantes consideran que los docentes deben utilizar herramientas de apoyo de simulación para sus clases teóricas, lo cual coincide 
con las comprobaciones de Ros y Conesa (2013), quienes indican que los profesores y tutores necesitan también un entrenamiento específico en el manejo, diseño e implementación de este tipo de acciones formativas utilizando la simulación. Igualmente hay que considerar en esta área que ambos grupos (experimental y control) manifestaron que no habían utilizado en otro curso de educación superior la metodología de simulación a través de un simulador como apoyo a su aprendizaje.

A la evaluación realizada al área de sentimientos de aproximación a los contenidos de la herramienta se obtuvo un porcentaje del $50 \%$ respecto a que les gustó la simulación al calmar el llanto del simulador; al 75\% no les gustó la simulación, por no poder cambiar fácilmente la ropa del simulador.

Además, las estudiantes detallaron algunas sugerencias para incorporar mejoras al simulador Baby Real Care II. Las listaron de acuerdo a sus preferencias, siendo la más recomendada "que se moje el pañal", "que la piel del Baby Real sea suave" y "que los ojos se abran y cierren" para hacer aún más realista la simulación.

Se concluyó que está demostrada la hipótesis, que la aplicación de la metodología de simulación con el uso del simulador difiere significativamente de la aplicación de la metodología tradicional. La metodología de simulación permite a los estudiantes experimentar situaciones idóneas para su ámbito profesional: les da la oportunidad de observar, analizar, repensar, retroalimentarse y repetir las actividades las veces que crean conveniente; las motiva a seguir perfeccionando sus destrezas y habilidades para desarrollar o mejorar las competencias propias de su campo laboral.

Los resultados expuestos brindan una alternativa a todos los profesionales que están inmersos en la educación superior, puedan utilizar en sus asignaturas esta metodología, pues les da la posibilidad de experimentar con casos reales en contextos simulados. Además les brinda la posibilidad de controlar la situación de acuerdo con los objetivos planteados y según lo que deseen perfilar en sus estudiantes como parte de sus competencias. También es preciso considerar, que esta genera- ción de estudiantes viven y se nutren de la tecnología; para ellos es más motivador el apropiarse de las TICs en sus sesiones de clases. Por esta razón, antes de aplicar la metodología de simulación, los docentes deben dominar el uso y aplicación del simulador como herramienta de apoyo en su asignatura.

\section{REFERENCIAS BIBLIOGRÁFICAS}

Aguirre, G. (2012). Postura experiencial de los docentes que utilizan la simulación clínica como estrategia didáctica en la carrera de Medicina. Tesis de maestría, Universidad Nacional de Colombia

Andrade, E. (2006). "Ambientes de Aprendizaje para la educación en Tecnología”. Recuperado de http://cvonline.uaeh. edu.mx/Cursos/Maestria/MTE/disenio_de_prog_de_amb_ de_apren/Unidad\%20II/amb_aprend_para_educ_tecnologica_Andrade.pdf

Andreu, A., García, M. y Mollar, M. (2005). La simulación y juego en la enseñanza-aprendizaje de lengua extranjera. Cuadernos Cervantes XI, 55 34-38.

Alvites-Huamaní, C. (2010). Evaluación a la Metodología de Simulación en la enseńanza aprendizaje de los cursos de Estimulación Temprana y Cuidados y protección del Infante. (Tesis de maestría). Universidad Nacional Mayor de San Marcos

Alvites-Huamaní, C. (2014) Simulation and Virtual Learning Environments: Tools for Teaching Psychology in Higher Education. Psychology Research, 4 (5) 376-382

Caride, J., Gradaille, R. y Teijeiro, J. (2012). El diálogo paradigmático en Educación Social: la simulación como un modo de enseñar y aprender en las aulas universitarias. Revista d'innovació educativa, 9, 23-32. Doi: 10.7203/attic.9.1959

Castro, S. (2008). Juegos, simulaciones y simulación-juego y los entornos multimediales en educación ¿mito o potencialidad? Revista de Investigación, 65, 223-245

Corvetto, M., Bravo, M., Montaña, Rodrigo, Utili, F., Escudero, E...Dagnino, J. (2013) Simulación en Educación médica una sinopsis, Revista Med Chile 141 70-79

Fernandez, R., Server, P. y Carballo, E. (2006) Aprendizaje con nuevas tecnologías paradigma emergente. ¿Nuevas modalidades de aprendizaje? Revista Electrónica de Tecnología Educativa, 20 (1) 1-24

Garcia-Carbonell, A., Watts, F. y Andreu, M. (2012) Simulación telemática como experiencia de aprendizaje de la lengua inglesa. Revista de Docencia Universitaria, 10 (3), 301-323

García, H., Navarro, L., López, M. y Rodríguez, F. (2014) Tecnologías de la información y la comunicación en salud y educación médica. Revista EDUMECENTRO, 6 (1) 253-265. Recuperado de http://scielo.sld.cu/scielo.php?pi- 


\section{$\mathrm{d}=$ S2077-8742014000100018\&script=sci_arttext}

Gisbert, M. (2013) Nuevos escenarios para aprendices digitales en la universidad. Revista de psicología Aloma, 31 (1) $55-64$

Ilia, G. (2000) Desarrollo de modelos y simuladores virtuales, Conferencia Simulation and Modeling Using Computers-Development Issues. Recuperado de www.psicol. unam.mx

Martínez, V., Martin, N. y Pérez, M. (s/f). El uso de las nuevas tecnologías para favorecer el trabajo en equipo. La simulación estratégica como técnica de aprendizaje experimental. Conocimiento, innovación y emprendedores: Camino al futuro.

Oleagordia, I. (2003) Estrategias educativas para el uso de las nuevas tecnologías de la Información y comunicación en la educación superior, Revista Iberoamericana, España

Orduz, R. (2012) Aprender y educar con las tecnologías del Siglo XXI, Corporación Colombia Digital.

Osorio, P., Ángel, M. y Franco, A. (2012) El uso de simuladores educativos para el desarrollo de competencias en la formación universitaria de pregrado. Revista Q Tecnología Comunicación Educación, 7 (13), 1-23

Pales, J. y Gomar, C. (2010) El uso de las simulaciones en educación médica, Revista TESI, 11 (2) 147-169

Ros, M. y Conesa, M. (2013) Adquisición de competencias a través de la simulación y juego de rol en el área contable. Revista Estudios sobre el mensaje periodístico 19 419428 DOI: http://revistas.ucm.es/index.php/ESMP/article/ view/42049

Realityworks (2006) Manual de instrucciones en español. Realityworks, Experiential Learning Technology. EE.UU. http://www.realityworks.com/products/realcare-baby

Salas, R. S. y Ardanza, P. (1995) La simulación como método de enseñanza y aprendizaje. Revista Educación Médica Superior 9, (1) 3-4 Recuperado http://scielo.sld.cu/scielo.php?script $=$ sci_arttext $\&$ pid $=$ S0864-21411995000100002\&ln$\mathrm{g}=\mathrm{es} \& \mathrm{nrm}=\mathrm{iso}$

Toledo, M. y Camero, R. (2010) Resultados preliminares de la aplicación de la simulación-juego instruccional (modificada): Viaje Intracelular. Revista de Investigación 34, 169-186 